\title{
Significados y Vivencias Subjetivas Asociados a la Presencia de un Trastorno de la Conducta Alimentaria, desde la Perspectiva de Mujeres que lo Padecen
}

\section{Subjective Life Experiences and Meanings Associated with the Presence of an Eating Disorder, from the Perspective of Women who Suffer from it}

\author{
Claudia Cruzat, Carolina Aspillaga, Maite Torres, Marcela Rodríguez, \\ Macarena Díaz y Constance Haemmerli \\ Universidad del Desarrollo
}

\begin{abstract}
El objetivo del estudio fue describir y relacionar las perspectivas, vivencias y teorías subjetivas de jóvenes chilenas con trastornos de la conducta alimentaria, respecto de su enfermedad. Se indagaron aspectos emocionales relacionados con las dinámicas internas, funcionamiento familiar, atribuciones causales y tratamiento. Se utilizó metodología cualitativa, con un diseño exploratorio y descriptivo. Se realizaron 8 entrevistas en profundidad y el análisis de los datos se basó en una codificación abierta, según los criterios de la Grounded Theory. Los resultados revelan que la enfermedad es vivida como un regulador de ansiedades y conflictos, que generaría tanto experiencias negativas como positivas, siendo estas últimas uno de los principales mantenedores del trastorno. Destacan los sentimientos de ambivalencia y cronicidad con que viven las pacientes su enfermedad.
\end{abstract}

Palabras clave: vivencias subjetivas, anorexia, bulimia, trastornos de la conducta alimentaria, tratamiento.

\begin{abstract}
The current study seeks to understand the different perspectives, life experiences and subjective theories of young Chilean women with an eating disorder regarding their condition, discerning emotional aspects related to family dynamics, causal attributions and treatment. Qualitative methodology with a descriptive-exploratory design is used. Eight in depth interviews were carried out. Data analysis was based on open coding in accordance with Grounded Theory. The findings reveal that the disorder is experienced as a regulator of conflict and anxiety, generating negative and positive experiences, these being one of the main reasons for maintaining the disorder. Feelings of ambivalence and chronicity are highlighted among patients living with this illness.
\end{abstract}

Keywords: subjective experiences, anorexia, bulimia, eating disorders, treatment.

\section{Introducción}

Los trastornos de la conducta alimentaria (TCA) son alteraciones de la conducta relacionadas con la ingesta, consecuencia de los esfuerzos por controlar el peso y la silueta. Según el DSM IV-TR, los TCA se dividen en anorexia nerviosa (AN), bulimia nerviosa (BN) y trastornos de la conducta alimentaria no especificados (TCANE) (American Psychiatric Association, 2002). Estos cuadros están estrechamente relacionados entre sí, compartiendo ciertos rasgos clínicos y observándose, en algunos casos, el cambio de uno a otro durante la evolución del desorden (Behar, 2004; Eddy, Dorer,

Claudia Cruzat Mandich, Facultad de Psicología, Universidad del Desarrollo, Santiago, Chile.

Carolina Aspillaga Hesse, Facultad de Psicología, Universidad del Desarrollo, Santiago, Chile.

Maite Torres Arrau, Facultad de Psicología, Universidad del Desarrollo, Santiago, Chile.

Marcela Rodríguez Rodríguez, Facultad de Psicología, Universidad del Desarrollo, Concepción, Chile.

Macarena Díaz Bugmann, Facultad de Psicología, Universidad del Desarrollo, Concepción, Chile.

Constance Haemmerli Delucchi, Facultad de Psicología, Universidad del Desarrollo, Santiago, Chile.

La correspondencia relativa a este artículo debe ser dirigida a Claudia Verónica Cruzat Mandich, Facultad de Psicología, Universidad del Desarrollo, Ainavillo 456, Concepción, Chile. E-mail: ccruzat@udd.cl

Esta investigación fue realizada gracias al apoyo financiero de la Dirección de Investigación de la Universidad del Desarrollo. 
Franko, Tahilani, Thompson-Brenner \& Herzog, 2008). En los tres tipos de trastorno siempre está presente el temor a engordar y la preocupación por la imagen corporal, lo que genera gran ansiedad (Marín, 2002).

De acuerdo a Cordella et al. (2006), uno podría preguntarse ¿cómo comprender una patología que parece generarse desde la voluntad? ¿por qué solo algunas mujeres se enferman, cuando tantas hacen dieta? ¿cómo se pueden reconocer los procesos de desarrollo que las dejan en riesgo, considerando que la mortalidad es tan alta y la calidad de vida tan mala?

Una intervención psicológica con pacientes con TCA es un desafío especial, ya que muchas pacientes están poco motivadas para cambiar sus hábitos alimentarios. Aunque el diagnóstico del subtipo, la edad, la duración de la enfermedad y los tratamientos previos no se han visto asociados con las fases de motivación, se plantea que las creencias que tiene la paciente acerca de su propia enfermedad se asocian directamente con la adherencia al tratamiento.

Dado este escenario, parece interesante describir la visión de las propias pacientes, en cuanto a la causa subjetiva de su enfermedad, el nivel de satisfacción con su proceso terapéutico y lo que a su juicio son considerados factores facilitadores de la recuperación y generadores de deserción.

\section{Antecedentes Teóricos y Empíricos}

\section{Principales Características de las Pacientes con TCA}

Se han determinado diversas características de las pacientes con TCA. Abraham, Von Lojewski, Anderson, Clarke y Russell (2009) exploraron los principales sentimientos que discriminan a las personas con TCA de las que no los padecen; haciendo referencia a distintas áreas: (1) aspectos psicológicos generales: las personas con TCA presentan mayor sensación de descontento y dificultad en las capacidades de afrontamiento, malestar en asistir a reuniones sociales y temor a la pérdida de control sobre las emociones; (2) conducta de comer: sienten incomodidad al ser vistas comien- do, preocupación por los alimentos y temor a la pérdida de control sobre el comer; y (3) imagen corporal: sensación de preocupación por la forma y peso corporal y temor a la pérdida de control sobre su cuerpo.

Las pacientes con TCA se caracterizan por presentar una baja conciencia de enfermedad. De acuerdo a las fases del modelo transteórico de Prochaska y DiClemente, las personas no cambian porque no pueden, no quieren o no saben cómo o qué cambiar (Prochaska \& Prochaska, 1999). Durante las entrevistas iniciales las pacientes aquejadas de AN se encuentran, generalmente, en un nivel de pre-contemplación (Fernández \& Turón, 2001); no se han propuesto cambiar porque sus síntomas son egosintónicos, no son conscientes aún de que constituyen un problema y más aún, un riesgo (Kirszman \& Salgueiro, 2002). En general, las pacientes con AN suelen ser llevadas a consulta obligadas por sus padres, presentando escasa o nula conciencia de enfermedad y negándose al cambio (Ward, Troop, Todd \& Treasure, 1996).

En el caso de las pacientes con $\mathrm{BN}$, la consulta suele ser más tardía debido a que tienen un peso cercano al normal, haciendo más difícil la detección. La mayoría acude a consultar cuando son sorprendidas en atracones o conductas compensatorias, reconociendo que existe un problema, pero sin darle la real importancia para seguir un tratamiento (Morandé, 1999), lo que podría corresponder a la etapa de contemplación, según el modelo transteórico del cambio.

Significados y Vivencias Subjetivas en TCA como Factor de Adherencia al Tratamiento

Krause (2005) señala que, para facilitar una comprensión más profunda de los cambios psicológicos que ocurren durante un proceso psicoterapéutico, debiera incluirse la vivencia que el propio consultante tiene de su proceso de cambio psicológico y de los elementos que lo hicieron posible, perspectiva que aún no ha sido suficientemente tratada en la investigación en psicoterapia.

Tomando en cuenta las características de estas pacientes, resulta importante describir la posición que estas tienen hacia su 
problema, considerando la motivación hacia el tratamiento, la conciencia de sí mismas y de su dolencia, la disponibilidad y el compromiso afectivo en el proceso terapéutico (Behar, 2004).

En investigaciones sobre la salud física se ha comprobado que las creencias que la gente tiene acerca de su enfermedad influyen en la capacidad para afrontar el tratamiento y en sus resultados. Este modelo de autorregulación también se está aplicando en salud mental y, al aplicarlo específicamente en la $\mathrm{AN}$, se supone que las creencias que estas pacientes tienen sobre su enfermedad estarían asociadas con la disposición al cambio (Higbed \& Fox, 2009).

Hill (2005) señala que la participación del cliente es una variable importante en el proceso de la terapia. Los clientes son agentes activos que deliberadamente buscan un tratamiento, se comprometen con el terapeuta y manejan con este las técnicas ofrecidas, excepto en los casos en que el tratamiento es obligado o forzado. Por lo tanto, un enfoque de tratamiento en el cual se reconoce y comprende la ambivalencia de estas pacientes puede ser más saludable, tanto para ellas como para los terapeutas (Treasure \& Ward, 1997). Asimismo, Treasure, Tchanturia y Schmidt (2005) señalan que es de suma importancia para los terapeutas tener una comprensión holística de este trastorno, que incorpore la visión de la propia paciente respecto de su enfermedad, los factores predisponentes y gatillantes, la asociación que hacen con el tema de la comida y sus motivaciones para mantener el TCA.

De acuerdo a lo anterior, parece pertinente evaluar el proceso terapéutico desde la perspectiva de las propias pacientes y describir, desde su propia visión, qué ha cambiado en ellas tras su tratamiento y cómo ha sido este proceso. Según Federici y Kaplan (2008), la motivación interna al cambio, la recuperación como un trabajo progresivo, el valor percibido de la experiencia de tratamiento, el desarrollo de relaciones de apoyo, la sensibilización y la tolerancia de las emociones negativas y auto-validación son puntos importantes a considerar en el tratamiento.

\section{Estudios sobre la Percepción que las Pacientes con TCA Tienen de su Enfermedad y Tratamiento}

Poco se sabe acerca de los ingredientes terapéuticos necesarios en el tratamiento para la recuperación de los TCA. Sorprendentemente, la mayoría de los estudios sobre este tema ha descuidado la opinión de la paciente. A continuación, se exponen los principales hallazgos que existen en la literatura acerca de los factores subjetivos que las pacientes con TCA atribuyen como causa de su enfermedad, los factores que a juicio de ellas son centrales para el logro de su recuperación y los factores que se relacionan con el nivel de satisfacción con el tratamiento.

En relación con los factores que las pacientes atribuyen como causales de su enfermedad, Tozzi, Sullivan, Fear, McKenzie y Bulik (2003) señalan que más de un tercio de las pacientes enfatiza la disfunción familiar como un factor contribuyente al desarrollo de su trastorno. Destacan la deprivación parental, el excesivo control, la poca relación con los padres, la tensión familiar y maltrato psicológico, como factores que conllevan a un alto nivel de estrés y frustración. Asimismo, Redenbach y Lawler (2003) encontraron que las mujeres con TCA mencionaban la falta de control de sus vidas y las expectativas poco realistas de la familia como razones para el desarrollo de su enfermedad. Relacionado con este hallazgo, Patching y Lawler (2009) señalan que el control, conexión y conflicto son tres aspectos centrales en el desarrollo y recuperación de un TCA. Las pacientes atribuyen el desarrollo de la enfermedad a la falta de control, la sensación de no conexión con la familia y compañeros y a graves conflictos con otras personas significativas.

$\mathrm{Al}$ investigar las opiniones de pacientes y ex pacientes con TCA acerca de su calidad de vida, las pacientes nombran con mayor frecuencia que las ex pacientes la psicopatología específica, reportando una pobre calidad de vida en la mayoría de los dominios, sobre todo en autoimagen y bienestar (De la Rie, Noordenbos, Donker \& Van Furth, 2007). 
Eivors, Button, Warner y Turner (2003) propusieron un estudio para investigar las posibles razones de los altos niveles de deserción, según las experiencias de las pacientes. El concepto de control surgió como central para la comprensión del proceso de deserción, destacando el carácter funcional de la enfermedad; para muchas se trata de un medio de supervivencia cuando se enfrentan con limitadas opciones para ir adquiriendo un sentido de control sobre su mundo.

Con respecto a los factores que las pacientes que padecen o han padecido un TCA atribuyen como centrales en la recuperación, se encuentran la autoconfianza y sentirse preparada y comprendida (Hsu, Crisp \& Callender, 1992). En la misma línea, Pettersen y Rosenvinge (2002) señalan que el deseo de cambiar, el tratamiento profesional, la atención no profesional y las personas significativas en las vidas de las pacientes son identificados como importantes factores de recuperación. Agregan una mejoría en la aceptación de sí misma, las relaciones interpersonales, la resolución de problemas y la satisfacción corporal. Asimismo, Lamoureux y Bottorff (2005) reportan que las mujeres que se han recuperado de $\mathrm{AN}$ señalan que la recuperación se centra en el redescubrimiento y la redefinición de sí mismas, incluyendo conocer los peligros, el avance progresivo, tolerar la exposición, las ventajas de una perspectiva distinta a la ideación anoréxica y el descubrimiento y recuperación de un sí mismo suficientemente bueno.

Federici y Kaplan (2008) identificaron, desde las perspectivas de las pacientes, ciertos factores que contribuyen a mantener un peso saludable y aquellos que intervienen en una recaída durante el período de seguimiento. Los análisis cualitativos revelaron seis principales categorías: motivación interna al cambio, recuperación como un trabajo progresivo, valor percibido de la experiencia de tratamiento, desarrollo de relaciones de apoyo, sensibilización $\mathrm{y}$ tolerancia de las emociones negativas y auto-validación. Según Patching y Lawler (2009), la recuperación se produce cuando las pacientes se vuelven a comprometer con la vida, desarrollando las competencias necesarias para la resolución de conflictos y redescubriendo el sentido de sí mismas, por lo que la recuperación estaría fuertemente relacionada con la autodeterminación y la autoaceptación.

Relacionado con lo anterior, De la Rie, Noordenbos, Donker y Van Furth (2008) investigaron la calidad del tratamiento de los TCA, desde la perspectiva de los terapeutas y pacientes, y compararon sus puntos de vista. Los resultados arrojaron que tanto terapeutas como pacientes mencionaban la alianza terapéutica y las habilidades comunicacionales como aspectos importantes en la calidad del tratamiento. Sin embargo, mientras los terapeutas les daban mayor valor a los síntomas y al cambio de comportamiento, las pacientes subrayaban la importancia de la relación terapéutica y el tratamiento de problemas subyacentes. Vanderlinden, Buis, Pieters y Probst (2007) consideran, además, como elementos fundamentales en el tratamiento de estas pacientes la mejora en la autoestima, la mejora de la experiencia corporal y el aprendizaje de habilidades de resolución de problemas.

En relación a los hallazgos que existen sobre la satisfacción de las pacientes con su tratamiento, Le Grange y Gelman (1998) evaluaron el punto de vista de las pacientes acerca de lo que es útil o perjudicial para su recuperación, luego de un año de terminar su tratamiento. Se encontró que la mayoría sentía que había mejorado, señalando como útil la psicoeducación, un entorno apoyador y el desafío a las creencias disfuncionales. El estudio de Rosenvinge y Klusmeier (2000) muestra que las pacientes estaban altamente satisfechas con la psicoterapia ambulatoria individual y grupal y, en menor medida, con la terapia familiar. Este hallazgo se relaciona con la investigación de Rorty, Yager y Rossotto (1993), en la que la mayoría de las mujeres informaron que sus madres y padres fueron más perjudiciales que útiles en el proceso de recuperación. Es importante destacar que la satisfacción con el tratamiento está fuertemente ligada a la percepción que las pacientes con TCA tienen de su terapeuta. Aquellas pacientes que percibían a su terapeuta como compe- 
tente en TCA reportaron mayor nivel de satisfacción con el tratamiento (Rosenvinge \& Klusmeier, 2000).

Finalmente, es relevante subrayar la idea expuesta por Patching y Lawler (2009), quienes plantean que no se debe entender el desarrollo y la recuperación de un TCA como eventos separados, sino como una sola entidad, como el viaje de un individuo tratando de descubrir y desarrollar el sentido de sí mismo.

\section{Objetivos y Relevancia del Estudio}

Pese a los hallazgos expuestos anteriormente, aún existen pocos estudios sobre este tema. Kelley (2005) plantea que las perspectivas y voces de las mujeres en terapia están en gran medida ausentes de la literatura, por lo que sería necesario comenzar a abordarlas.

La descripción y comprensión de la percepción que tienen las propias pacientes acerca de su enfermedad y tratamiento son consideradas un gran aporte en el proceso terapéutico, ya que orientan el abordaje del tratamiento, brindando un mejor pronóstico.

Dado este escenario, surgió la pregunta que guió la presente investigación: ¿Cuáles son los significados y vivencias subjetivas asociados a la presencia de TCA, desde la perspectiva de mujeres que los padecen? Para responder a esta pregunta fue necesario abordar los aspectos emocionales, los elementos relacionados con el tratamiento, las dinámicas y funcionamiento familiar y las posibles causas asociadas a los trastornos alimentarios.

\section{Método}

\section{Diseño}

Debido a la búsqueda de los significados $\mathrm{y}$ vivencias subjetivas que las pacientes con TCA le otorgan a su enfermedad, el método cualitativo era la herramienta metodológica que mejor se ajustaba a la interrogante de la investigación. Se utilizó un diseño exploratorio, descriptivo y relacional. Esta investigación se basó en los supuestos planteados por Glaser y Strauss (1967) para la recolección y análisis de datos, de la escuela teórica Grounded Theory.

\section{Instrumento}

Se utilizó la entrevista en profundidad, definida por Taylor y Bogdan (1998) como un encuentro cara a cara entre el investigador y el o los informantes, dirigida hacia la comprensión de las perspectivas que tienen respecto de sus vidas y experiencias, tal como las expresan en sus propias palabras. La entrevista se realizó en base a un guión temático, diseñado a partir de las preguntas directrices de la investigación.

\section{Estrategia de Muestreo y Características de la Muestra}

El muestreo fue inicialmente intencionado y luego teóricamente guiado, según los resultados y categorías emergentes en el análisis de los datos. Los criterios de inclusión en la muestra fueron: participantes mujeres, adolescentes o jóvenes, que padecieran o hubieran padecido de TCA y que hubieran recibido o estuvieren en tratamiento psicoterapéutico individual para tratar su enfermedad. El criterio de exclusión fue que las participantes se encontraran en la fase inicial de su tratamiento, debido a la baja conciencia de enfermedad que tienen en ese momento, ya que la realización de la entrevista podría interferir negativamente y a que se tendría poca información respecto de las vivencias asociadas al proceso terapéutico.

La muestra quedó constituida por ocho mujeres solteras, entre 13 y 29 años, todas con diagnóstico de AN y algunas también con sintomatología bulímica. Del total de entrevistadas, la mitad presentaba síntomas y se encontraba actualmente en tratamiento; la otra mitad había sido dada de alta. El nivel socioeconómico de la mayoría de las pacientes era medio-alto y vivían con alguno de sus padres, exceptuando una entrevistada que se encontraba en un hogar de protección y que pertenecía a un nivel socioeconómico bajo. Seis de las entrevista- 
das eran hijas mayores y en cinco casos sus padres estaban separados.

Si bien la aspiración inicial era contar con una muestra constituida por adolescentes que presentaran actualmente sintomatología, las dificultades para obtener participantes que accedieran a colaborar en la investigación limitó las posibilidades de una muestra más homogénea y obligó a flexibilizar los criterios de edad y el estado de desarrollo de la enfermedad. Sin embargo, esto también enriqueció los resultados, ya que permitió conocer la perspectiva que tenían sobre su tratamiento las pacientes que estaban dadas de alta y que ya no presentaban sintomatología.

Las estrategias de muestreo utilizadas fueron la técnica de bola de nieve (Polit \& Hungler, 2000), siendo las mismas participantes quienes derivaron a siguientes entrevistadas, y el uso de informantes claves que, en este caso, fueron sus terapeutas.

\section{Procedimiento}

Una vez realizado el primer contacto con las participantes y que estas accedieron a ser parte del estudio, se contactó a sus terapeutas (en el caso de quienes estaban en tratamiento) para pedirles su autorización para realizar las entrevistas, con el propósito de evitar que estas pudiesen ser perjudiciales para las pacientes.

Anterior a la realización de la entrevista, las pacientes firmaron un consentimiento informado en donde se les explicaban las características del estudio, sus fines, el anonimato de sus respuestas y la posibilidad de abandonar la investigación en caso que quisieran hacerlo.

Las entrevistas las realizaron las psicólogas miembros del equipo de investigación. En ellas participó una sola entrevistadora con el objetivo de generar un espacio menos amenazante y de mayor intimidad para las entrevistadas. Las entrevistas fueron realizadas en los domicilios de las participantes o en las dependencias de la consulta de sus terapeutas. Tuvieron una duración aproximada de noventa minutos y fueron grabadas en formato de audio.

Cada entrevista fue transcrita íntegramente, procediéndose a su análisis, el cual fue simultáneo a la recolección de datos, con el fin de realizar un muestreo teóricamente guiado.

\section{Análisis de Datos}

Para el análisis de datos se usó codificación abierta, axial y selectiva (Mella, 2003; Rodríguez, Gil \& García, 1999), con el fin de describir y relacionar conceptos, generando un modelo descriptivo y relacional que permita un mejor entendimiento de los TCA, desde la visión de pacientes con este diagnóstico.

Se mantuvieron presentes criterios de rigurosidad para mantener los estándares de cientificidad de la investigación. En primer lugar, el trabajo de campo está completamente documentado. Además, se utilizó la triangulación de investigadores en el análisis de los datos, pues fue realizado en equipo con el fin de alcanzar el criterio de intersubjetividad. Para contribuir a la validez interna, se utilizó el criterio de credibilidad, mediante la confirmación de los hallazgos y la revisión de algunos datos específicos por parte de las informantes, con el objetivo de que pudieran corregir los errores de interpretación de los hechos. El contacto permanente con los terapeutas permitió también contrastar y corroborar hallazgos.

\section{Resultados}

A continuación se presentan los resultados a nivel descriptivo, en base a la realización de una codificación abierta de los datos, y a nivel analítico relacional, obtenidos luego de la realización de la codificación axial y selectiva.

Presentar los resultados descriptivos tiene por objetivo lograr que el lector comprenda, desde la información más concreta y ejemplificadora, cómo se fue desarrollando el modelo que explica las principales vivencias que las entrevistadas tienen en torno a su TCA. Estos resultados descriptivos fueron organizados en base a cinco categorías principales que surgieron desde el relato de las participantes.

En la Tabla 1 se presentan las categorías, junto con sus subcategorías. 
Tabla 1

Categorías y Subcategorías Elaboradas según el Contenido de las Entrevistas

\begin{tabular}{|c|c|c|}
\hline Categorías & Subcategorías & Contenidos \\
\hline \multirow{7}{*}{$\begin{array}{l}\text { 1. Factores percibidos } \\
\text { como gatillantes de la } \\
\text { enfermedad }\end{array}$} & - Factores ambientales & - Cánones de belleza \\
\hline & & - Valoración social del control alimentario \\
\hline & - Factores familiares & $\begin{array}{l}\text { - Ambiente familiar que presionaría a ser } \\
\text { "perfecta" }\end{array}$ \\
\hline & & - Madres que dan relevancia al peso \\
\hline & - Aspectos individuales & - Autoexigencia \\
\hline & & - Necesidad de atención \\
\hline & & - Baja autoestima \\
\hline \multirow{5}{*}{$\begin{array}{l}\text { 2. Vivencias asociadas a la } \\
\text { presencia del TCA }\end{array}$} & - Nivel cognitivo & $\begin{array}{l}\text { - Comida y peso como eje central del } \\
\text { pensamiento }\end{array}$ \\
\hline & & - Falsa conciencia de enfermedad \\
\hline & - Nivel emocional & $\begin{array}{l}\text { Emociones positivas (agrado, rol } \\
\text { regulador) }\end{array}$ \\
\hline & & $\begin{array}{l}\text { Emociones negativas (angustia, tristeza, } \\
\text { desesperanza) }\end{array}$ \\
\hline & - Nivel social & - Actitud de evasión (incomodidad y temor) \\
\hline \multirow{2}{*}{$\begin{array}{l}\text { 3. Factores asociados a la } \\
\text { mantención del TCA }\end{array}$} & - Rol de regulador emocional & - TCA como "adicción y necesidad" \\
\hline & - Vivencias emocionales negativas & - Angustia, tristeza y desesperanza \\
\hline \multirow{4}{*}{$\begin{array}{l}\text { 4. Vivencias de las pacientes } \\
\text { con respecto a la terapia }\end{array}$} & - Experiencia terapéutica durante & - Como obstáculo de objetivos \\
\hline & la enfermedad & - Falsa conciencia de enfermedad \\
\hline & $\begin{array}{l}\text { - Experiencia terapéutica una vez } \\
\text { superada la enfermedad }\end{array}$ & $\begin{array}{l}\text { - Vínculo con el terapeuta como facilitador } \\
\text { de cambio }\end{array}$ \\
\hline & & $\begin{array}{l}\text { - Importancia de la toma de conciencia de } \\
\text { la enfermedad }\end{array}$ \\
\hline \multirow{6}{*}{$\begin{array}{l}\text { 5. Aspectos percibidos como } \\
\text { facilitadores de la cura }\end{array}$} & - Apoyo del entorno & - Autonomía \\
\hline & - Proceso Terapéutico & - Guía \\
\hline & & $\begin{array}{l}\text { - Fuente de herramientas para mejorar la } \\
\text { relación con sí misma y el entorno }\end{array}$ \\
\hline & - Cambio a nivel personal & $\begin{array}{l}\text { - Toma de conciencia de enfermedad y sus } \\
\text { riesgos }\end{array}$ \\
\hline & & $\begin{array}{l}\text { - Responsabilidad en el tratamiento e } \\
\text { intención de cambiar }\end{array}$ \\
\hline & & - Metas y proyecto de vida \\
\hline
\end{tabular}




\section{Resultados Descriptivos}

Factores percibidos como gatillantes de la enfermedad. Las pacientes asocian como elementos influyentes en el desarrollo de su TCA a factores ambientales, familiares e individuales.

Respecto a los factores ambientales, perciben que la valoración social del control alimentario pudo influir en el desarrollo de su enfermedad, así como los cánones de belleza asociados a la delgadez.

En cuanto a los factores familiares, destacan la percepción de un ambiente familiar exigente que las presionaría a ser "perfectas". Enfatizan que para sus madres el peso es un tema relevante, lo que pudo influir en la presión que sienten por ser delgadas. La siguiente cita permite comprender lo señalado:

Yo siempre me iba a Cachagua donde siempre era todo muy bonito, muy perfeccionista, veraneaba con mis primas, las mellizas, que eran regias, $y$ por ese lado había un rasgo de que yo tenía que ser perfecta. Por otro lado, mi mamá siempre me... como que me tenía el parámetro muy alto, de que yo tenía que ser bonita, yo tenía que ser flaca, yo tenía que hablar bien, yo tenía que tener las mejores amigas, si no me iba bien en el colegio, tenía que ser educadita, comer bien, siempre bien princesita... (Entrevista 4, párrafos 10 y 11)

Las entrevistadas también mencionan determinados conflictos familiares como significativos en el desarrollo del trastorno; específicamente, refieren haber recibido poca atención por parte de sus familiares.

Por último, respecto de los aspectos individuales y de personalidad, las entrevistadas destacan la autoexigencia, la necesidad de llamar la atención y su baja autoestima.

Vivencias asociadas a la presencia del $T C A$. Esta categoría se puede dividir en vivencias cognitivas, emocionales y sociales.

A nivel cognitivo, la comida y el peso se convierten, durante la enfermedad, en el eje del pensamiento de las jóvenes. A su vez, presentan una falsa conciencia de enfermedad, la que se evidencia en que solo se perciben enfermas a nivel racional, ya que, si bien tienen conocimiento del daño que se pueden ocasionar, sus deseos de adelgazar son más intensos:

... yo siento que la enfermedad, ni siquiera la percibo como una enfermedad porque no logré el fin que quería. Si yo me hubiese visto como moribunda, en los huesos, bueno, estuve enferma, tuve bulimia... (Entrevista 8, párrafo 21)

A nivel emocional, coexisten emociones negativas y positivas, relacionándose estas últimas con los factores que ayudarían a mantener el trastorno. Las vivencias negativas hacen referencia a sentimientos de angustia y desesperanza, vinculados a pensamientos de no poder controlar la situación en que se encuentran, percibiendo que esta será crónica en sus vidas. Las pacientes oscilan entre los polos de obsesión y perfeccionismo a inestabilidad y descontrol. Señalan restringirse en aspectos como la alimentación pero, a su vez, refieren ser impulsivas y muy lábiles. Además, señalan presentar ánimo depresivo, que se manifestaría en tristeza, sentimientos de soledad, anhedonia y aplanamiento afectivo.

Entre las vivencias positivas asociadas al trastorno, destaca el rol de regulador emocional que este cumple. Las conductas alimentarias tendrían la función de manejar y canalizar sus emociones, permitiéndoles recibir el alivio, la tranquilidad y el placer que no les es posible obtener por otros medios. Otra vivencia positiva es la intensa sensación de agrado experimentada frente a la pérdida de peso, sintiéndose más atractivas y valoradas por su ambiente.

A nivel social, existiría una fuerte preocupación respecto de cómo son percibidas por los demás, lo que las llevaría a mantener una actitud de evasión, originada por su incomodidad al comparar constantemente su cuerpo con los estándares de cuerpo ideal construidos por la sociedad. Temen al rechazo, a que los demás se den cuenta de sus conductas alimentarias y a la idea de ser presionadas por los demás para que coman o no vomiten.

Mantención del trastorno. Entre los elementos percibidos como influyentes en la mantención del trastorno, destaca el sentir 
que restringirse en la alimentación y/o tener atracones y vomitar les genera adicción y necesidad. Esto se relacionaría con el rol de regulador emocional que cumplirían sus conductas alimentarias. La siguiente cita da cuenta de la función que cumple la comida en sus vidas:

...es mi... no sé, es como mi... mi... mi desahogo... es como una droga pa' un adicto es como un, es como la forma en que yo puedo desahogar mis emociones, no puedo expresarla de otra manera. Es como... si estoy feliz, puta quiero comer, toda mi vida gira en torno a la comida, todo, todo, todo. (Entrevista 7, párrafo 56).

A su vez, las vivencias emocionales negativas, como la angustia, la tristeza y la desesperanza, también les impedirían mejorarse y salir del estado en que se encuentran.

Relación con el propio cuerpo durante el TCA. Durante la enfermedad, el peso posee un fuerte significado emocional, hay desconexión con las sensaciones del organismo y el cuerpo es vivido como fuente de tensión. Las entrevistadas simbolizan la delgadez como sinónimo de seguridad, perfección y felicidad. Por el contrario, la gordura es percibida como incomodidad, asco e infelicidad. De este modo, solo sienten agrado cuando se ven delgadas, manifestando insatisfacción con su propio cuerpo y un fuerte temor a subir de peso. Este temor a engordar las lleva a cierto ascetismo, manifestado en no prestar atención a sensaciones corporales básicas, como el hambre.

Experiencia terapéutica. Con respecto a las vivencias terapéuticas durante el transcurso de la enfermedad, se evidencia en las pacientes una falsa conciencia de enfermedad, que se expresa en considerar que están en terapia por temas distintos al trastorno alimentario que padecen. Otro aspecto es la comparación de su cuerpo con el de su terapeuta y la percepción de la terapia como un impedimento para lograr su meta de adelgazar.
En relación a la vivencia terapéutica luego de superada la enfermedad, las entrevistadas que estaban dadas de alta perciben que la terapia fue generadora de cambio y el vínculo con el terapeuta es valorado positivamente como motor de este. Refieren que el proceso terapéutico las hizo tomar conciencia de que estaban enfermas, les permitió querer mejorarse, conocerse, entender y reconciliarse con el entorno y mejorar su autoestima.

En cuanto al vínculo con el terapeuta como facilitador del cambio, explicitan que la relación cálida y cariñosa que se establece, la disposición del terapeuta hacia ellas, la percepción de ser aceptadas y la preocupación sincera fueron claves para la superación de su enfermedad.

Aspectos percibidos como facilitadores de la cura. Las entrevistadas que estaban dadas de alta describen tres grandes aspectos que facilitaron su proceso de cura: (a) el apoyo del entorno más cercano, destacando que no las trataran como incapacitadas y se les diera autonomía; (b) el proceso terapéuti$c o$, señalando que fue una guía que las ayudó a mejorar, entregándoles las herramientas para entenderse, mirarse, centrarse en sí mismas y reconciliarse con el entorno; y c) el cambio a nivel personal, destacando la toma de conciencia de su enfermedad, percibir el riesgo que esta conllevaba, sentirse responsables de su tratamiento y tener ganas de mejorar. Además, consideran que plantearse metas y tener un proyecto de vida concreto como, por ejemplo, querer ser profesionales y tener hijos, las ayudó a generar este cambio. Esto ocurrió en la medida en que se dieron cuenta de que el TCA les impediría lograr estos sueños.

\section{Resultados Analíticos Relacionales}

A partir de los resultados descritos anteriormente, se generó un modelo explicativo, basado en la vinculación que existe entre las distintas categorías. Este modelo pretende lograr una comprensión relacional de las vivencias asociadas a la presencia de un trastorno alimentario (ver Figura 1). 


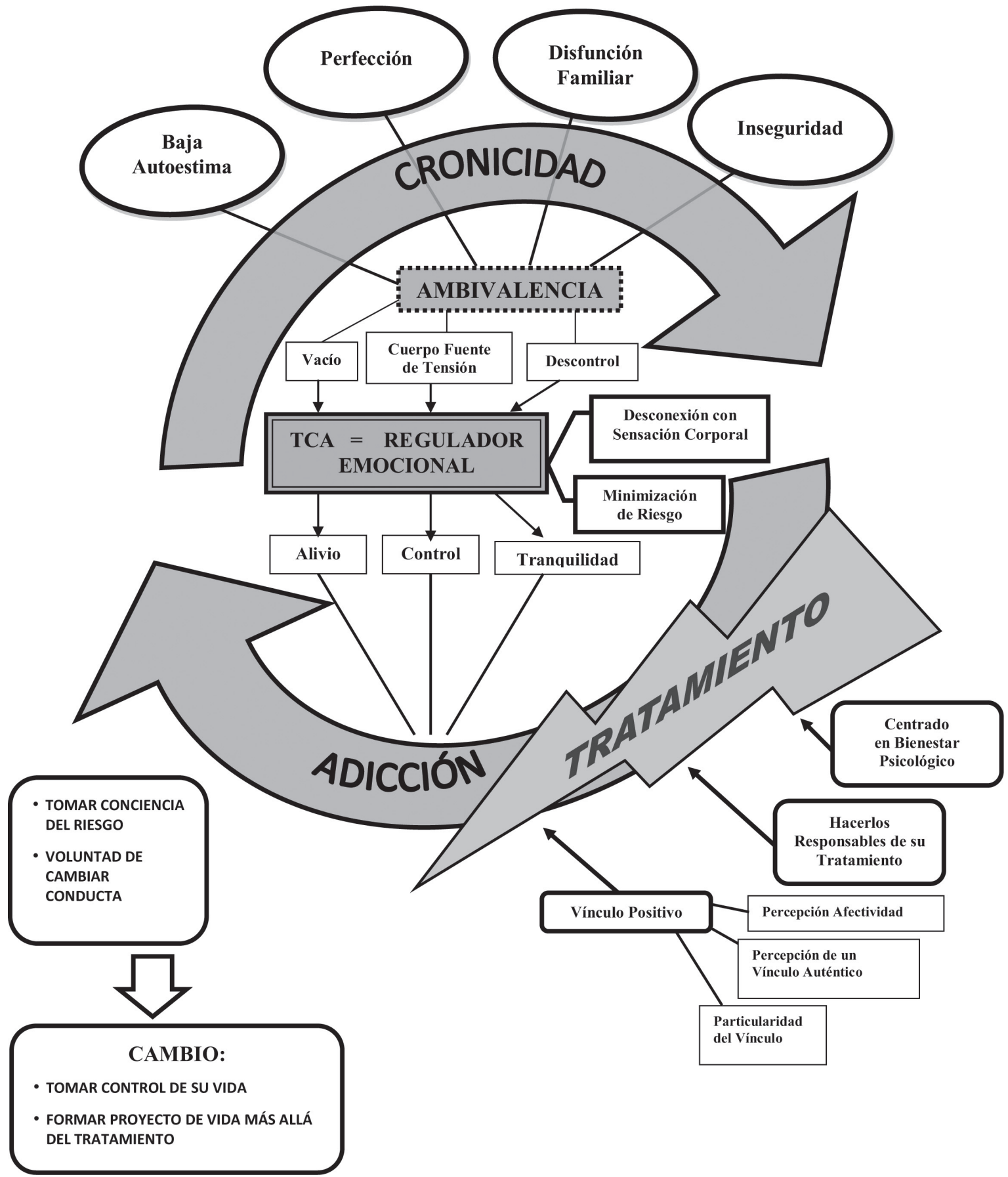

Figura 1. Trastorno de la conducta alimentaria como regulador emocional.

De acuerdo a los resultados obtenidos, los TCA tendrían un origen multicausal, basado en factores familiares, sociales y personales que se interrelacionarían entre sí. En sus familias, las pacientes percibi- rían alteraciones de distintos tipos e intensidades, como conflictos, daños o falta de cuidado. Las jóvenes sentirían una presión social y familiar por ser perfectas que, conjugado con su baja autoestima e inseguri- 
dad, las llevaría a sentir que deben tener un cuerpo perfecto para ser aceptadas y valoradas.

En este deseo y necesidad de alcanzar la perfección corporal, el TCA pasaría a convertirse en un regulador de sus emociones negativas. Las pacientes sentirían que su único mecanismo para manejarlas consiste en restringir su ingesta de alimentos y/o tener atracones de comida, lo que les generaría sensaciones de alivio, control y tranquilidad. Sin embargo, por otro lado, esta misma conducta las llevaría a desarrollar sentimientos negativos, como sensación de vacío, descontrol y a significar su cuerpo como fuente de tensión. De este modo, los TCA no solo se relacionarían con querer tener el cuerpo soñado, sino que serían un mecanismo para regular las emociones negativas, puesto que no cuentan con otra herramienta para hacerles frente. Esto llegaría a tal extremo que las pacientes se desconectarían de la percepción de sus sensaciones fisiológicas y tendrían una mínima conciencia de daño que, si está presente, solo es a nivel cognitivo. La coexistencia de emociones negativas, manejadas por medio del TCA, y las vivencias positivas asociadas a los efectos de esta regulación generarían en las pacientes sentimientos de ambivalencia. Si bien tienen una conciencia racional del daño que se provocan, no desearían modificar estas conductas porque no sabrían como manejar sus emociones. Como resultado de esto se incrementarían, a través de la ambivalencia, sus emociones negativas.

Creer que el único mecanismo regulador que poseen contra sus ansiedades, temores y descontroles es la conducta alimentaria alterada, las llevaría a sentirse adictas a esta, evidenciándose un deseo racional de cambiarla, pero, al mismo tiempo, la necesidad de repetirlas y mantenerlas. Estas percepciones y vivencias de ser adictas al TCA las llevarían a vivir su enfermedad como si se tratase de una situación crónica, ante la cual no tienen esperanzas de recuperarse, pero, por otra parte, tampoco desean realmente cambiar.

La terapia vendría a generar un quiebre de este círculo vicioso, provocado por la emociones positivas asociadas al TCA y a su rol regulador, y permitiría romper este sistema. Sin embargo, los requisitos que debiera cumplir la terapia para lograrlo son: a) centrarse en el bienestar psicológico (más allá de la conducta alimentaria específica); b) permitir que las pacientes se hagan cargo y se sientan responsables de su tratamiento y proceso terapéutico; y c) desarrollar un vínculo terapéutico que permita una vivencia de afectividad, se perciba autenticidad en la relación y en el que haya una particularidad, es decir, las jóvenes sientan que no son solo una paciente más. Todos estos elementos conjugados llevarían a las pacientes a mejorar su autoestima y a elaborar sus inseguridades y emociones negativas, desarrollando, a su vez, nuevas estrategias y mecanismos de enfrentamiento. Así se sentirían más fuertes emocionalmente y con control sobre ellas mismas.

La ruptura del círculo vicioso de cronicidad y adicción, por medio del tratamiento, generaría como consecuencia la toma de conciencia del riesgo asociado a la sintomatología alimentaria y, por otra parte, el surgimiento de la voluntad de cambiar la conducta. Este hecho sería central, ya que en las entrevistas se visualizó que el cambio solo comienza a aparecer cuando las pacientes deciden comenzar a hacerlo. Dicho cambio implicaría una toma de control de sus propias vidas (tener proyectos centrados en ellas mismas y en su desarrollo personal) y no en la necesidad de ser perfectas para ser aceptadas en su medio.

\section{Discusión y Conclusiones}

Los principales hallazgos de este estudio dan cuenta de la fuerte carga emocional con que viven las pacientes con trastornos alimentarios. El peso y el cuerpo se convierten en el eje de sus pensamientos, cumplen el rol de regulador emocional y están investidos de fuertes significados para ellas. El peso ocuparía un rol tan central en sus vidas que las jóvenes evitarían situaciones sociales para no ser cuestionadas por sus conductas alimentarias. A su vez, su obsesión las haría vivir en una constante comparación de su cuerpo con el de otros, lo que les provocaría gran incomodidad. 
El cuerpo y la belleza física estarían investidos de una fuerte carga emocional. La delgadez representaría el polo de la felicidad, el éxito y la seguridad, mientras que la gordura sería el polo de la infelicidad, lo indeseado y lo desagradable. Los cambios de peso se vivirían de forma intensa, sintiendo pánico frente a la posibilidad de engordar. Las jóvenes desarrollarían una desconexión con sus sensaciones corporales y fisiológicas, distorsionando tanto la interpretación del hambre y la saciedad como la percepción de su propia imagen, elementos que limitan notablemente las posibilidades de tener conciencia de enfermedad y de los riesgos de sus conductas alimentarias.

Según los resultados señalados en esta investigación, emocionalmente el TCA generaría tanto experiencias negativas como positivas: las primeras aludirían a sentimientos de desesperanza, ánimo depresivo y a la oscilación entre obsesión y perfeccionismo e inestabilidad y descontrol; las vivencias positivas aludirían a la regulación emocional y a percibirse más atractivas y valoradas. Es así como la enfermedad es vivenciada por las pacientes como un regulador de ansiedades y conflictos, generándose una necesidad de mantener la conducta alimentaria para manejar las emociones negativas. Esta situación es vivida como adictiva y la paciente, pese a tener conciencia cognitiva de daño, no puede ni desea modificar sus hábitos. Ello se relaciona con la percepción de las jóvenes de la cronicidad de la enfermedad, lo que no les permite imaginarse la vida sin el trastorno, lo cual generaría sentimientos de desesperanza.

A nivel del tratamiento de los TCA, estos resultados permiten explicar ciertas conductas, como la resistencia al cambio y la negación de la enfermedad, logrando así comprender que las jóvenes no desean modificar sus conductas alimentarias porque no conocen otras vías para manejar sus emociones, así como por las emociones positivas que el TCA les genera.

Los resultados confirman la multicausalidad de los TCA asociada a aspectos sociales, familiares y personales. Las pacientes destacan como facilitadores de la génesis del trastorno un medio social y familiar exigente, en donde se espera perfección en el ámbito físico y en donde el peso es un tema particularmente relevante para sus madres. Las pacientes perciben que el trastorno ha sido, inconscientemente, un llamado de atención hacia sus familias, de quienes sienten no haber recibido los cuidados requeridos para desarrollarse sanamente. A nivel personal, las jóvenes refieren como elementos influyentes ser autoexigentes y tener una baja autoestima.

En cuanto a los factores facilitadores de la cura, existen elementos externos e internos que las pacientes relatan como influyentes. Entre los factores externos, se encuentra la terapia psicológica y la contención de familiares y amigos. Entre los aspectos individuales, las pacientes señalan el logro de una transformación interna que las llevaría a mejorar su autoestima, a querer sanar, a tomar real conciencia de riesgo y a plantearse metas y proyectos mas allá de su peso. En el desarrollo de esta transformación la terapia tendría un rol central, en la que la percepción de un vínculo positivo con el terapeuta sería valorada como motor de cambio. De los aspectos relevantes del vínculo destacan aquellos que tienen que ver con la relación y no aquellos que aluden a las técnicas usadas por los terapeutas.

Citando a Sevillano (2001), a la hora de iniciar el tratamiento con estas pacientes, hay que tomar en cuenta tres aspectos fundamentales: a) conciencia del trastorno, b) relación terapéutica y c) motivación y expectativas de cambio. Estos aspectos son relevantes, ya que pueden determinar en gran medida las posibilidades de aceptación del tratamiento y el cambio en sus conductas alteradas.

Tal como lo plantean las pacientes, la autoestima tiene un rol central en el trastorno, tanto en su inicio como en su resolución. La enfermedad en sí y la investidura positiva que dan a la belleza y a la delgadez serían mecanismos para reforzar su autoestima, de modo que el tratamiento debiera dar centralidad a este tema, ya que las pacientes perciben que cuando mejoró su autoestima se generó un cambio en ellas que les permitió querer mejorar, plantearse metas y tomar el control de sus 
vidas. Lo anterior concuerda con el estudio de Vanderlinden et al. (2007), que considera como elementos fundamentales en el tratamiento de estas pacientes la mejora en la autoestima, la mejora de la experiencia corporal y el aprendizaje de habilidades de resolución de problemas.

Por otra parte, el proceso terapéutico es vivido, durante el período crítico del TCA, con una "falsa conciencia de enfermedad", que parece más bien un discurso aprendido, influenciado por deseabilidad social. En esta etapa creen que la terapia no surge como respuesta al trastorno, sino como una instancia que busca resolver otras temáticas de conflicto en sus vidas, vivenciando el tratamiento como un impedimento para alcanzar el logro de sus metas.

Entre los principales resultados encontrados en las jóvenes que han superado sus síntomas, se aprecia que significan el tratamiento como una experiencia de aprendizaje que les sirvió para valorarse, conocerse y generar nuevas estrategias de enfrentamiento al estrés. Actualmente, buscan sentirse cómodas consigo mismas y señalan ser precavidas, prestando atención a sus conductas alimentarias. Considerando lo anterior, sería importante incluir elementos de autovalía, autoconocimiento y estrategias de afrontamiento como aspectos centrales en el mantenimiento de logros y prevención de recaídas.

En relación a las limitaciones de este estudio, el principal problema radicó en la dificultad de acceso a la muestra. Dadas las características de este trastorno, respecto de la conciencia de enfermedad y resistencia al cambio, si estas pacientes no manifiestan interés en sus procesos terapéuticos, menos aún lo tienen en colaborar con una investigación. Lo anterior generó la necesidad de flexibilizar los criterios de inclusión, ampliándose el rango de edad de las entrevistadas e incluyendo, no solo a pacientes que se encontraran padeciendo la sintomatología del trastorno sino, también, a jóvenes que ya no presentaban síntomas y habían finalizado su tratamiento. Sin embargo, esto permitió obtener información inicialmente no planificada, referida a las vivencias que las pacientes tienen de la enfermedad, desde la mirada de quienes ya no presentarían síntomas y la habrían superado. Esto permitió describir y relacionar las vivencias asociadas al TCA y a su tratamiento, tanto en el momento en que se está padeciendo como cuando se ha podido superar la enfermedad, permitiendo comprender el trastorno tanto en su inicio, evolución y tratamiento, como en su superación.

$\mathrm{Al}$ analizar los resultados, surge como interrogante principal: ¿cuáles serán aquellas habilidades requeridas en el terapeuta para establecer un vínculo profundo con el paciente que padece TCA y que favorecen la mejoría de los síntomas? Considerando la importancia que las pacientes otorgan al vínculo terapéutico en su tratamiento, también sería de gran utilidad realizar estudios sobre la relación entre la alianza terapéutica y la efectividad en los resultados de la psicoterapia en pacientes con TCA.

Junto a lo anterior, aparece como relevante considerar el carácter adictivo que tiene la enfermedad, por lo que sería interesante incluir en su tratamiento elementos específicos de las terapias que trabajan con pacientes adictos.

Dado el rol que las pacientes atribuyen a sus madres en el origen del trastorno, sería relevante estudiar a las madres de jóvenes con TCA para mostrar cómo se relacionan con el cuerpo, el peso, la belleza física y cómo vivencian y significan la enfermedad de sus hijas.

\section{Referencias}

Abraham, S., Von Lojewski, A., Anderson, G., Clarke, S. \& Russell, J. (2009). Feelings: What questions best discriminate women with and without eating disorders? (Abstract). Eating and Weight Disorders, 14, e6-e10. Extraído el 5 julio, 2009, de http://www.kurtis.it/home/en/abstract. cfm?articolo_id $=6166 \&$ CFID

American Psychiatric Association (2002). DSM IV-TR Manual diagnóstico y estadístico de los trastornos mentales: texto revisado. Barcelona: Masson.

Behar, R. (2004). Trastornos de la conducta alimentaria: clínica y epidemiología. En R. Behar \& G. Figueroa (Eds.), Anorexia nerviosa y bulimia. Clínica y terapéutica (pp. 17-53). Santiago, Chile: Mediterráneo.

Cordella, P., Lizana, P., Urrejola, P., Figueroa, D., del Río, P., Hodgson, I. et al. (2006). Programa de trastornos de alimentación en adolescentes y jóvenes chilenos: variaciones a seis meses de tratamiento. Revista Médica de Chile, 134, 973-980. 
De la Rie, S., Noordenbos, G., Donker, M. \& Van Furth, E. (2007). The patient's view on quality of life and eating disorders. International Journal of Eating Disorders, 40, 13-20.

De la Rie, S., Noordenbos, G., Donker, M. \& Van Furth, E. (2008). The quality of treatment of eating disorders: A comparison of the therapists' and the patients' perspective. International Journal of Eating Disorders, 41, 307-317.

Eddy, K. T., Dorer, D. J., Franko, D. L., Tahilani, K., Thompson-Brenner, H. \& Herzog, D. B. (2008). Diagnostic crossover in anorexia nervosa and bulimia nervosa: Implications for DSM-V (Versión electrónica). The American Journal of Psychiatry, $165,245-250$.

Eivors, A., Button, E., Warner, S. \& Turner, K. (2003). Understanding the experience of drop-out from treatment for anorexia nervosa. European Eating Disorders Review, 11, 90-107.

Federici, A. \& Kaplan, A. S. (2008). The patient's account of relapse and recovery in anorexia nervosa: A qualitative study. European Eating Disorders Review, 16, 1-10.

Fernández, F. \& Turón, V. (2001). Trastornos de la alimentación: guía básica de tratamiento en anorexia y bulimia. Barcelona: Masson.

Glaser, B. G. \& Strauss, A. L. (1967). The discovery of Grounded Theory. Chicago: Aldine Publishing Company.

Higbed, L. \& Fox, J. R. E. (2009). Illness perceptions in anorexia nervosa: A qualitative investigation (Abstract). British Journal of Clinical Psychology, July 3. Extraído el 5 Julio, 2009, de http://www. ingentaconnect.com/content/bpsoc/bjcp/preprints/bjcp772

Hill, C. E. (2005). Therapist techniques, client involvement, and the therapeutic relationship: Inextricably intertwined in the therapy process. Psychotherapy: Theory, Research, Practice, Training, 42, 431-442.

Hsu, L. K. G., Crisp, A. H. \& Callender, J. S. (1992). Recovery in anorexia nervosa: The patient's perspective. International Journal of Eating Disorders, 11, 341-350.

Kelley, J. P. (2005). What do women in therapy for an eating disorder find helpful? A qualitative study. Dissertation Abstracts International. Section B. The Sciences and Engineering, 65, 6274.

Kirszman, D. \& Salgueiro, M. (2002). El enemigo en el espejo. De la insatisfacción corporal al trastorno alimentario. Madrid: TEA Ediciones.

Krause, M. (2005). Psicoterapia y cambio. Una mirada desde la subjetividad. Santiago, Chile: Ediciones Universidad Católica de Chile.

Lamoureux, M. M. H. \& Bottorff, J. L. (2005). "Becoming the real me": Recovering from anorexia nervosa (Abstract). Health Care for Women International, 26, 170-188. Extraído el 5 Julio, 2009, de http:// www.informaworld.com/smpp/content $\sim \mathrm{db}=$ all?co ntent $=10.1080 / 07399330590905602$

Le Grange, D. \& Gelman, T. (1998). Patients' perspective of treatment in eating disorders: A preliminary study. South African Journal of Psychology, 28(3), 182-186.

Marín, V. (2002). Trastorno de la conducta alimentaria en escolares y adolescentes. Revista Chilena de Nutrición, 29, 86-91.

Mella, O. (2003). Metodología cualitativa en ciencias sociales y educación. Orientaciones teóricometodológicas y técnicas de investigación. Santiago, Chile: Primus.

Morandé, G. (1999). La anorexia. Cómo combatir y prevenir el miedo a engordar en las adolescentes. Madrid: Temas de Hoy.

Patching, J. \& Lawler, J. (2009). Understanding women's experiences of developing an eating disorder and recovering: A life-history approach (Versión electrónica). Nursing Inquiry, 16, 10-21.

Pettersen, G. \& Rosenvinge, J. H. (2002). Improvement and recovery from eating disorders: A patient perspective. Eating Disorders: The Journal of Treatment \& Prevention, 10, 61-71.

Polit D. F. \& Hungler B. P. (2000). Diseño y métodos en la investigación cualitativa. En D. F. Polit \& B. $\mathrm{P}$. Hungler, Investigación científica en ciencias de la salud ( $6^{\mathrm{a}}$ ed., pp. 231-247). Ciudad de México: McGraw-Hill Interamericana.

Prochaska, J. O. \& Prochaska, J. M. (1999). Why don't continents move? Why don't people change? Journal of Psychotherapy Integration, 9, 83-102.

Redenbach, J. \& Lawler, J. (2003). Recovery from disordered eating: What life histories reveal (Abstract). Contemporary Nurse, 15, 148-156. Extraído el 6 Mayo, 2009, de http://www.ncbi. nlm.nih.gov/pubmed/14649520

Rodríguez, G., Gil, J. \& García, E. (1999). Métodos de investigación cualitativa. En G. Rodríguez, J. Gil \& E. García, Metodología de la investigación cualitativa (pp. 39-59). Granada: Ediciones Aljibe.

Rorty, M., Yager, J. \& Rossotto, E. (1993). Why and how do women recover from bulimia nervosa? The subjective appraisals of forty women recovered for a year or more. International Journal of Eating Disorders, 14, 249-260.

Rosenvinge, J. H. \& Klusmeier, A. K. (2000). Treatment for eating disorders from a patient satisfaction perspective: A Norwegian replication of a British study. European Eating Disorders Review, 8, 293300.

Sevillano, J. P. (2001). Evaluación y motivación en los trastornos de la conducta alimentaria. En E. García-Camba, Avances en trastornos de la conducta alimentaria. Anorexia nerviosa, bulimia nerviosa, obesidad (pp. 75-92). Barcelona: Masson.

Taylor, S. J. \& Bogdan, R. (1998). Introducción a los métodos cualitativos de investigación. Buenos Aires: Paidós.

Tozzi, F., Sullivan, P. F., Fear, J. L., McKenzie, J. \& Bulik, C. M. (2003). Causes and recovery in anorexia nervosa: The patient's perspective. International Journal of Eating Disorders, 33, 143-154.

Treasure, J., Tchanturia, K. \& Schmidt, U. (2005). Developing a model of the treatment for eating disorder: Using neuroscience research to examine the how rather than the what of change. Counselling \& Psychotherapy Research, 5, 191202. 
Treasure, J. \& Ward, A. (1997). A practical guide to the use of motivational interviewing in anorexia nervosa. European Eating Disorders Review, 5, 102-114.

Vanderlinden, J., Buis, H., Pieters, G. \& Probst, M. (2007). Which elements in the treatment of eating disorders are necessary 'ingredients' in the recovery process? A comparison between the patient's and therapist's view. European Eating Disorders Review, 15, 357-365.

Ward, A., Troop, N., Todd, G. \& Treasure, J. (1996). To change or not to change. 'How' is the question? British Journal of Medical Psychology, 69, 139146.

Fecha de recepción: Junio de 2009.

Fecha de aceptación: Marzo de 2010. 